\title{
Intracerebral Hemorrhage Secondary to Ventriculoperitoneal Shunt Insertion -Four Case Reports-
}

\author{
Kouichi MISAKI, Naoyuki UCHIYAMA, \\ Yutaka HAYASHI, and Jun-ichiro HAMADA \\ Department of Neurosurgery, Graduate School of Medical Science, \\ Kanazawa University, Kanazawa, Ishikawa
}

\begin{abstract}
Four patients presented with intracerebral hemorrhage secondary to ventriculoperitoneal (VP) shunt insertion. VP shunt insertion was performed for idiopathic normal-pressure hydrocephalus (Case 1), hydrocephalus after cerebellar hemorrhage (Case 2), and subarachnoid hemorrhage followed by meningitis (Cases 3 and 4). Cerebral hemorrhage was confirmed 4 hours (Case 1), 2 days (Case 2), 7 days (Case 3 ), and 13 days (Case 4) after the operation. Cases 1 and 2 required intraoperative hemostasis for bleeding from the cortical vein. The 7 previous and our 4 patients were divided according to early (within 2 days after shunt placement, $n=6$, Group 1) and delayed (5-13 days post-shunting, $n=5$, Group 2) hemorrhage. Bleeding was attributable to venous occlusion due to intraoperative manipulation in Group 1, and to the vulnerability of brain tissue induced by a primary brain disease in Group 2.
\end{abstract}

Key words: intracerebral hemorrhage, ventriculoperitoneal shunt, hydrocephalus, complication, ventricular catheter 


\section{Introduction}

Postoperative complications after ventriculoperitoneal (VP) shunt placement include shunt obstruction, subdural hematoma, infection, seizures, catheter migration, and shunt malfunction. ${ }^{1,9)}$ Intracerebral or intraventricular hemorrhage secondary to VP shunt placement is uncommon. Only 7 patients have been reported with this complication, and retrospective analysis revealed an incidence rate of $0.3-4.0 \% .^{1,2,5,7,8)}$ The features of this complication are not clearly understood.

Here we report 4 additional patients with cerebral hemorrhage after ventricular shunt procedure among 215 shunt procedures performed between 1998 and 2008 $(1.9 \%)$.

\section{Case Reports}

All patients underwent placement of a VP shunt system connected to a CODMAN HAKIM programmable valve with an antisiphon device (Medos S.A., Le Locle, Switzerland). The programmed valve pressure was 150, 50, 70, and $60 \mathrm{mmH}_{2} \mathrm{O}$ in Cases 1-4, respectively. A ventricular catheter was inserted into the lateral ventricle through a frontal or occipital burrhole after test tapping. The burrhole was located at the coronal suture at the mid-papillary line for frontal access to the ventricle or approximately $5 \mathrm{~cm}$ above the inion and $6 \mathrm{~cm}$ lateral from the midline for occipital access. None of the patients required more than 3 attempts, and no blood was observed in the cerebrospinal fluid at cannulation of the ventricles. None of the 4 patients presented with evidence of coagulopathy or bleeding tendency, or was receiving anti-platelet or anticoagulant medication.

Case 1: An 82-year-old hypertensive woman had a 10month history of gait disturbance, dementia, and urinary incontinence. Computed tomography (CT) of the head showed moderately enlarged lateral ventricles with an Evans index of $31.4 \%$. Under a diagnosis of idiopathic normal-pressure hydrocephalus, a VP shunt was placed via a right frontal horn puncture. Intraoperative bleeding from the cortical vein under the exposed dura was relatively strong, so another burrhole was needed for ventricular catheter cannulation. She experienced generalized convulsion 4 hours after the operation. Head CT showed a $4.8 \times 4.7 \mathrm{~cm}$ subcortical hematoma close to the ventricular catheter (Fig. 1A, B). We did not treat the hematoma surgically. $T_{2}$-weighted magnetic resonance (MR) imaging before the operation showed no evident organic lesions such as vascular malformation or brain tumor. $\mathrm{T}_{2}^{*}$-weighted MR imaging was not performed.

Case 2: An 80-year-old woman with a history of atrial fibrillation and chronic heart failure developed hydrocephalus 4 weeks after removal of cerebellar hemorrhage. She underwent VP shunt placement through a right frontal burrhole. Hemostasis was required to stop intraoperative bleeding from the cortical vein. Head CT performed shortly after the operation showed no hematoma along the shunt tube (Fig. 1C). She suddenly developed

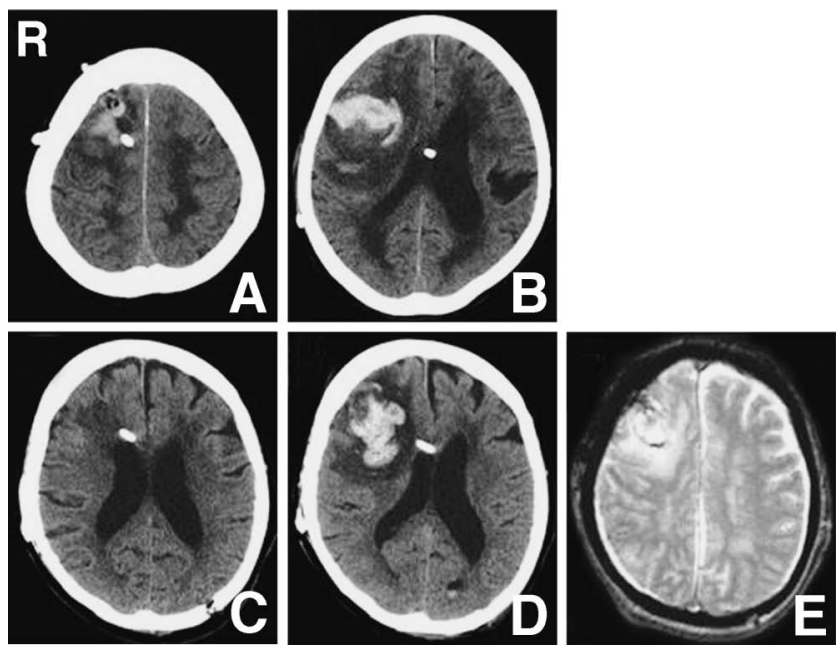

Fig. 1 A, B: Case 1. Computed tomography (CT) scans of the head obtained 4 hours after shunt placement demonstrating a subcortical hematoma around the shunt tube. C-E: Case 2. CT scan obtained just after shunt placement (C) does not depict the intracerebral hematoma that was detected 2 days later (D). Postoperative $T_{2}^{*}$-weighted magnetic resonance image (E) showing no evidence of microbleeds.

consciousness disturbance and left-sided hemiparesis on the 2 nd postoperative day. CT demonstrated a $4.7 \times 2.8$ cm intraparenchymal hemorrhage (Fig. 1D). Postoperative $\mathrm{T}_{2}^{*}$-weighted gradient-echo imaging disclosed no evident microbleeds (Fig. 1E) and other MR imaging showed no remarkable organic lesion around the hematoma.

Case 3: A 55-year-old man without previous diseases had suffered subarachnoid hemorrhage ( $\mathrm{SAH}$ ) due to rupture of a right internal carotid-posterior communicating artery aneurysm which was clipped. The subsequent hydrocephalus was treated with a VP shunt in the right anterior horn of the lateral ventricle. Two years later, he was hospitalized again with meningitis and shunt malfunction. The infected shunt system was removed and another shunt was introduced after he recovered from meningitis. A ventricular tube was placed in the left posterior horn of the lateral ventricle. CT obtained immediately after the operation demonstrated no hematoma, but routine followup CT performed on the 7 th postoperative day disclosed a $2.8 \times 1.3 \mathrm{~cm}$ subcortical hematoma (Fig. 2A, B). Preoperative $\mathrm{MR}$ imaging including $\mathrm{T}_{2}^{*}$-weighted sequences revealed no microbleeds or other organic lesions (Fig. 2C).

Case 4: A 64-year-old man with treated hypertension was admitted to our hospital with SAH. A ruptured anterior communicating artery aneurysm was clipped via the left pterional approach. Postoperatively he developed meningitis attributable to ventricular drainage through the left frontal burrhole and a VP shunt was placed 4 weeks after aneurysm clipping. The shunt catheter was inserted in the right posterior horn of the lateral ventricle. The operative procedure was uneventful, and head CT performed 6 days later disclosed normalization of the ventricular size without hematoma. CT acquired 13 days after the operation revealed a subcortical $2.0 \times 1.7 \mathrm{~cm}$ hematoma along 

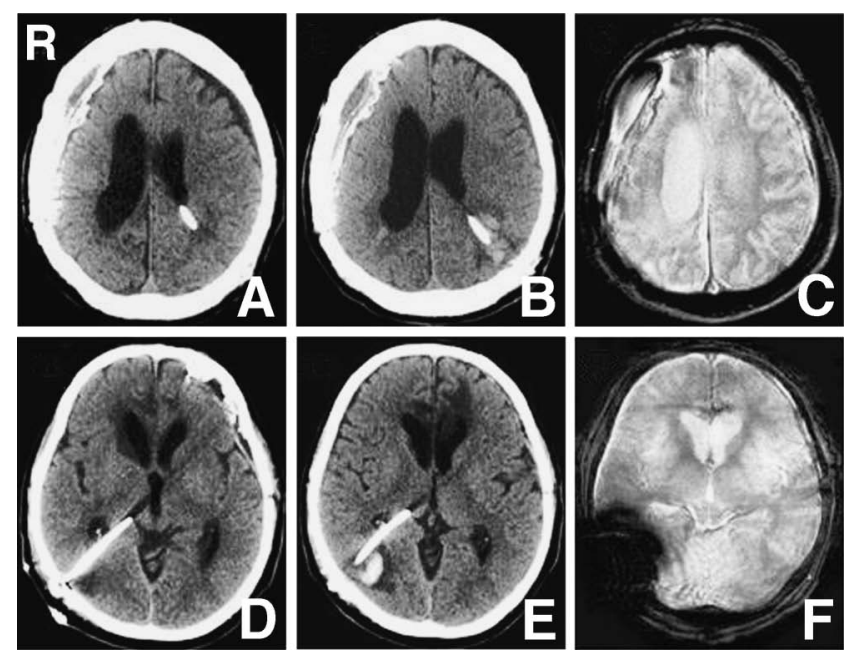

Fig. 2 A-C: Case 3. Computed tomography (CT) scan of the head acquired shortly after shunt placement (A) showing no subcortical hematoma. CT scan obtained 7 days after the procedure (B) depicting a hematoma surrounding the shunt tube. $\mathrm{T}_{2}^{*}$-weighted magnetic resonance (MR) image obtained before shunt insertion (C) revealing no signal intensity loss indicative of microbleeds. D-F: Case 4. CT scans acquired 6 (D) and 13 days (E) postoperatively demonstrating a subcortical hematoma. Postoperative $T_{2}^{*}$-weighted MR image (F) showing no evidence of microbleeds. the catheter, but no neurological deficits (Fig. 2D, E). No obvious microbleeds or lesions were detected by postoperative MR imaging including $\mathrm{T}_{2}^{*}$-weighted gradient echo sequences (Fig. 2F).

\section{Discussion}

Intracerebral hemorrhage after ventricular cannulation may result from coexistent bleeding disorder, shunt-induced disseminated intravascular coagulation, disruption of an intracerebral tumor, hemorrhage from an occult vascular malformation, or head trauma shortly after shunt placement. ${ }^{7,8)}$ MR imaging showed that none of our 4 patients manifested evidence of bleeding disorders or organic lesions. None of the 7 previous patients with postoperative intracerebral hemorrhage around the shunt tube presented with evidence of any of the above risk factors. ${ }^{1,2,5,7,8)}$ We divided the 7 previous and our 4 patients into 2 groups based on whether the hemorrhage occurred early (within 2 days of VP shunting, $\mathrm{n}=6$, Group 1 , Table 1) or late (5-13 days after VP shunting, $\mathrm{n}=5$, Group 2, Table 2).

Hemostasis of bleeding from a cortical vein was difficult in 2 of the 6 patients in Group 1. Another patient in Group 1 had undergone a $1-\mathrm{cm}^{3}$ cortical biopsy for pathological confirmation of Alzheimer's disease followed by hemostasis of the biopsy site prior to shunt insertion. ${ }^{2}{ }^{2}$ Therefore, disturbances in venous return due to hemostasis of a cortical vein may result in early subcortical hemorrhage after shunt placement.

Table 1 Cases of early intracerebral hemorrhage after ventriculoperitoneal shunt insertion

\begin{tabular}{|c|c|c|c|c|c|}
\hline \multirow{2}{*}{ Series } & \multirow{2}{*}{$\begin{array}{l}\text { Age (yrs)/ } \\
\text { Sex }\end{array}$} & \multirow{2}{*}{ Primary disease } & \multicolumn{2}{|r|}{ Shunt operation } & \multirow{2}{*}{$\begin{array}{l}\text { Onset day of } \\
\text { hemorrhage }\end{array}$} \\
\hline & & & Site & Operative finding & \\
\hline \multirow[t]{2}{*}{ Derdeyn et al. (1988) ${ }^{2)}$} & $56 / \mathrm{M}$ & ASDH & $\mathrm{PH}$ & uneventful & 0 \\
\hline & $73 / \mathrm{F}$ & iNPH & $\mathrm{AH}$ & brain biopsy before shunting & 0 \\
\hline \multirow[t]{2}{*}{ Savitz and Bobroff (1999) ${ }^{7)}$} & - & - & $\mathrm{PH}$ & - & $0-2$ \\
\hline & - & - & $\mathrm{PH}$ & - & $0-2$ \\
\hline Case 1 & $82 / \mathrm{F}$ & iNPH & $\mathrm{AH}$ & bleeding from cortical vein & 0 \\
\hline Case 2 & $80 / \mathrm{F}$ & cerebellar hemorrhage & $\mathrm{AH}$ & bleeding from cortical vein & 2 \\
\hline
\end{tabular}

AH: anterior horn of lateral ventricle, ASDH: acute subdural hemorrhage, iNPH: idiopathic normal pressure hydrocephalus, PH: posterior horn of lateral ventricle, —: data unavailable.

Table 2 Cases of delayed intracerebral hemorrhage after ventriculoperitoneal shunt insertion

\begin{tabular}{|c|c|c|c|c|c|}
\hline \multirow{2}{*}{ Series } & \multirow{2}{*}{$\begin{array}{l}\text { Age (yrs)/ } \\
\text { Sex }\end{array}$} & \multirow{2}{*}{ Primary disease } & \multicolumn{2}{|c|}{ Shunt operation } & \multirow{2}{*}{$\begin{array}{l}\text { Onset day of } \\
\text { hemorrhage }\end{array}$} \\
\hline & & & Site & Operative finding & \\
\hline Matsumura et al. $(1985)^{5)}$ & $17 / \mathrm{M}$ & brain contusion & $\mathrm{AH}$ & uneventful & 7 \\
\hline Snow et al. $(1986)^{8)}$ & $43 / \mathrm{F}$ & iNPH & $\mathrm{AH}$ & uneventful & $5-7$ \\
\hline Alcazar et al. $(2007)^{1)}$ & $64 / \mathrm{F}$ & $\mathrm{SAH}$ & $\mathrm{PH}$ & uneventful & 6 \\
\hline Case 3 & $55 / \mathrm{M}$ & SAH, meningitis & $\mathrm{PH}$ & uneventful & 7 \\
\hline Case 4 & $64 / \mathrm{M}$ & $\mathrm{SAH}$, meningitis & $\mathrm{PH}$ & uneventful & $6-13$ \\
\hline
\end{tabular}

AH: anterior horn of lateral ventricle, iNPH: idiopathic normal pressure hydrocephalus, $\mathrm{PH}$ : posterior horn of lateral ventricle, SAH: subarachnoid hemorrhage. 
The VP shunts were placed after clipping of a ruptured aneurysm and recovery from subsequent meningitis in 2 of the 5 patients in Group 2. Delayed hemorrhage occurred after shunt placement for hydrocephalus secondary to $\mathrm{SAH}$ in one patient, and delayed hemorrhage 2 years manifested after ipsilateral traumatic contusional hematoma leading to recent memory disturbance and epilepsy in another. ${ }^{1,5)}$ Therefore, 4 of the 5 patients in Group 2 presented with a primary brain disorder such as $\mathrm{SAH}$, meningitis, or severe brain trauma prior to shunt placement. We suggest that tube cannulation into fragile brain tissue affected by a primary brain disorder was associated with the delayed development of an intracerebral hematoma.

The mechanism underlying the development of a delayed intraparenchymal hematoma after shunt placement may involve erosion of a cerebral blood vessel secondary to contact with the ventricular catheter, ${ }^{1)}$ or a similar mechanism. ${ }^{8)}$ However, this mechanism fails to explain all post-shunting hemorrhages because continuous contact must also occur between the ventricular catheter and blood vessels in many patients without this complication. We consider fragile brain tissue due to a primary disorder as well as disruption of a blood vessel by the catheter as the main factors that contribute to delayed cerebral hemorrhage after shunt placement.

Fragility of the brain is not easy to detect on brain images. The microbleed confirmed on $\mathrm{T}_{2}^{*}$-weighted MR imaging, reflecting hemosiderin deposits, is related to advanced age and hypertension, and is a risk factor for subsequent intracerebral hemorrhage. ${ }^{3,6)}$ Histological analysis after surgical resection identified several fragile arteries surrounding the microbleeds, suggesting that such vessels reflect the fragility of brain tissue. ${ }^{4)}$ None of 3 of our 4 patients investigated by $\mathrm{T}_{2}^{*}$-weighted MR imaging showed evidence of microbleeding. Studies of larger patient populations are needed to ascertain the correlation between microbleeds and cerebral hemorrhage following VP shunt placement.

In conclusion, intracerebral hemorrhage secondary to VP shunt placement is a complication rather than an accident. The large number of shunt surgeries and the small number of reported patients with this complication suggests that many cases with this complication are not reported. The present cases suggest that the risk factors for early and delayed post-shunting hemorrhage are different. We suggest that early hemorrhage is attributable to venous occlusion due to intraoperative manipulation whereas delayed bleeding occurs in fragile brain tissue compromised by an existing brain disorder.

\section{References}

1) Alcazar L, Alfaro R, Tamarit M, Gomez-Angulo JC, Ortega JM, Aragones P, Jerez P, Salazar F, del Pozo JM: Delayed intracerebral hemorrhage after ventriculoperitoneal shunt insertion. Case report and literature review. Neurocirugia (Astur) 18: 128-133, 2007

2) Derdeyn CP, Delashaw JB, Broaddus WC, Jane JA: Detection of shunt-induced intracerebral hemorrhage by postoperative skull films: A report of two cases. Neurosurgery 22: 755-757, 1988

3) Imaizumi T, Honma T, Horita Y, Kawamura M, Kohama I, Miyata K, Nyon KS, Niwa J: The number of microbleeds on gradient $\mathrm{T} 2{ }^{*}$-weighted magnetic resonance image at the onset of intracerebral hemorrhage. J Stroke Cerebrovasc Dis 17: 30-34, 2008

4) Kikuta K, Takagi Y, Nozaki K, Okada T, Hashimoto N: Histological analysis of microbleed after surgical resection in a patient with moyamoya disease - case report. Neurol Med Chir (Tokyo) 47: 564-567, 2007

5) Matsumura A, Shinohara A, Munekata K, Maki Y: Delayed intracerebral hemorrhage after ventriculoperitoneal shunt. Surg Neurol 24: 503-506, 1985

6) Naka H, Nomura E, Takahashi T, Wakabayashi S, Mimori Y, Kajikawa H, Kohriyama T, Matsumoto M: Combinations of the presence or absence of cerebral microbleeds and advanced white matter hyperintensity as predictors of subsequent stroke types. AJNR Am J Neuroradiol 27: 830-835, 2006

7) Savitz MH, Bobroff LM: Low incidence of delayed intracerebral hemorrhage secondary to ventriculoperitoneal shunt insertion. J Neurosurg 91: 32-34, 1999

8) Snow RB, Zimmerman RD, Devinsky O: Delayed intracerebral hemorrhage after ventriculoperitoneal shunting. Neurosurgery 19: 305-307, 1986

9) Wu Y, Green NL, Wrensch MR, Zhao S, Gupta N: Ventriculoperitoneal shunt complications in California: 1990 to 2000. Neurosurgery 61: 557-562, 2007

Address reprint requests to: Kouichi Misaki, M.D., Department of Neurosurgery, Graduate School of Medical Science, Kanazawa University, 13-1 Takara-machi, Kanazawa, Ishikawa 920-8641, Japan.

e-mail: misaki@ns.m.kanazawa-u.ac.jp 